\title{
Carcinoid heart disease from ovarian primary presenting with acute pericarditis and biventricular failure
}

\author{
D Vergani, L Massironi, F Lombardi, C Fiorentini
}

Division of Cardiology, San Paolo Hospital, University of Milan, Milan, Italy

D Vergani

L Massironi

F Lombardi

C Fiorentini

Correspondence to: Dr D Vergani, v. Anguissola 31, 20146 Milano, Italy.

Accepted for publication 8 June 1998

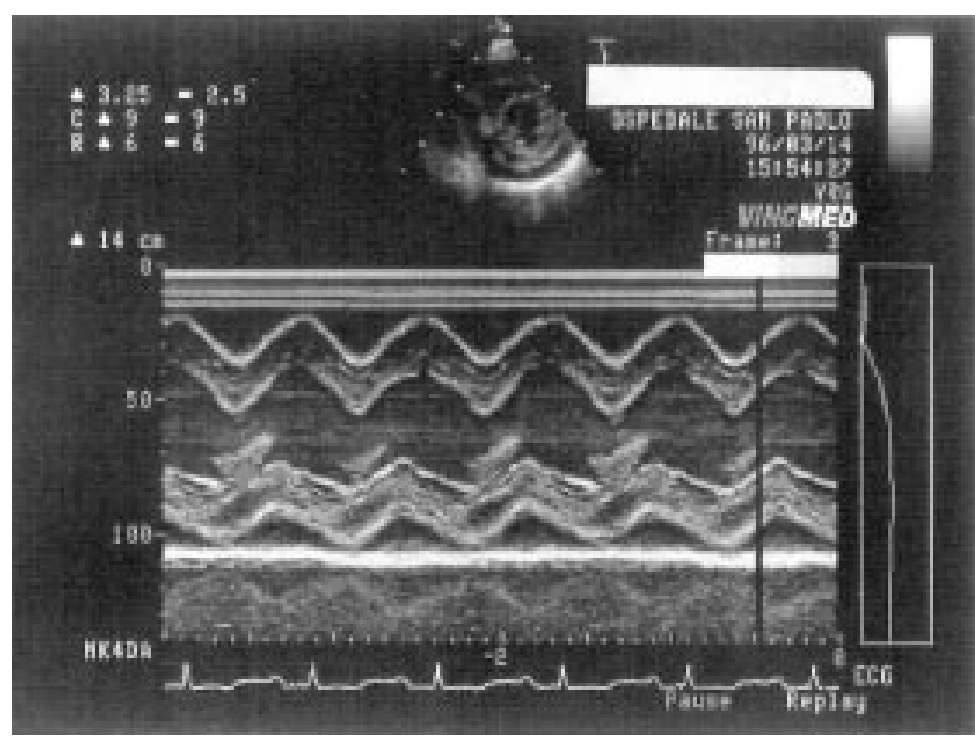

Figure 1 Echocardiogram on first admission. A large pericardial effusion is seen around the cardiac chambers with a "swinging heart" appearance.

\begin{abstract}
A case is described of a 54 year old woman who had acute pericarditis with large exudative effusion accompanied by severe right and left ventricular failure. The patient was finally diagnosed with carcinoid heart disease from an ovarian carcinoid teratoma. She was treated with octreotide-a somatostatin analoguefollowed by radical surgical resection of the neoplasm. At one year follow up only mild carcinoid tricuspid regurgitation remained. Only 16 cases of carcinoid heart disease from an ovarian primary have been described in literature. Moreover clinically manifest acute, nonmetastatic pericarditis and left heart failure are not considered as possible presentations of carcinoid heart disease, whatever the origin. In a recent series a small pericardial effusion was considered an infrequent and unexpected echocardiographic finding in carcinoid heart patients. One case of "carcinoid pericarditis" has previously been described as a consequence of pericardial metastasis. Left sided heart involvement is
\end{abstract}

\section{A 54 rear old}

A 54 year old woman was admitted to our division complaining of a dry, irritating cough and slight evening fever for 15 days and more recent mild dyspnoea and dull chest pain. She was in physiological menopause and a check up three years ago had excluded cardiovascular disease. A mild, grade $2 / 6$ holosystolic murmur could be heard on the left lower sternal border and pericardial friction rubs persisted for a few days after admission. The liver was palpable three fingerbreadths below the right costal margin. Prominent jugular veins, abdominal-jugular reflux, and mild bilateral ankle oedema could be appreciated. ECG showed non-specific ST-T changes. Raised serum lactic dehydrogenase and aminotransferase were found on admission; creatine kinase was repeatedly normal. Chest radiography showed pronounced enlargement of the cardiac silhouette and normal lungs. Echocardiography showed a large pericardial effusion without signs of cardiac tamponade (fig 1), moderate to severe tricuspid regurgitation with right atrial enlargement, and reduced left ventricular systolic function. 
A

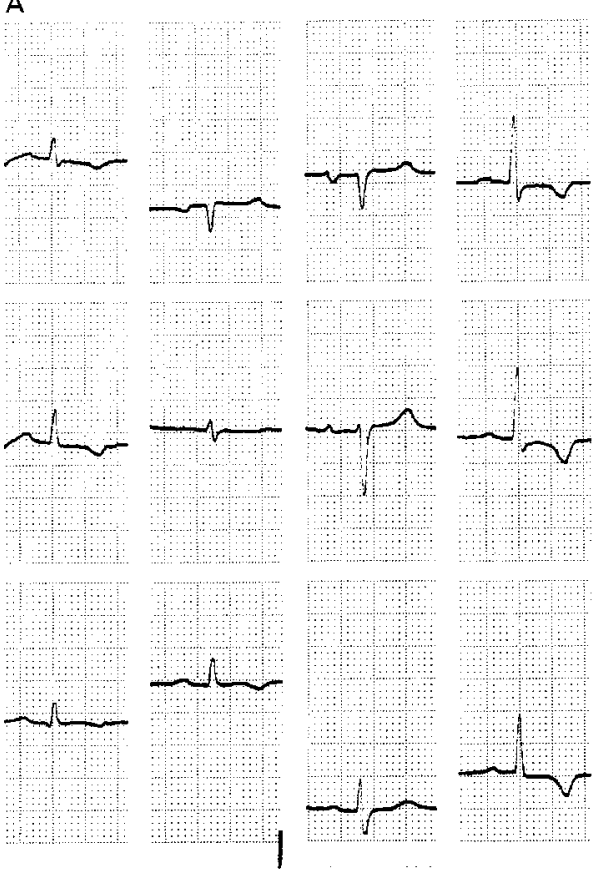

B

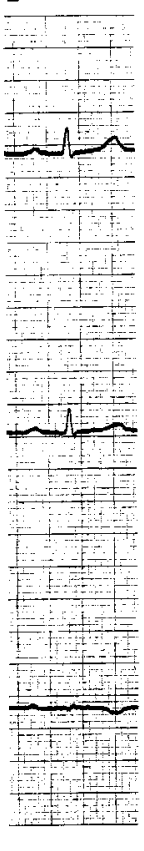

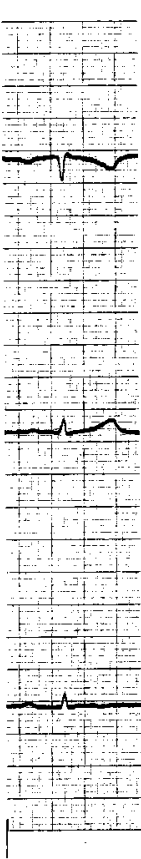
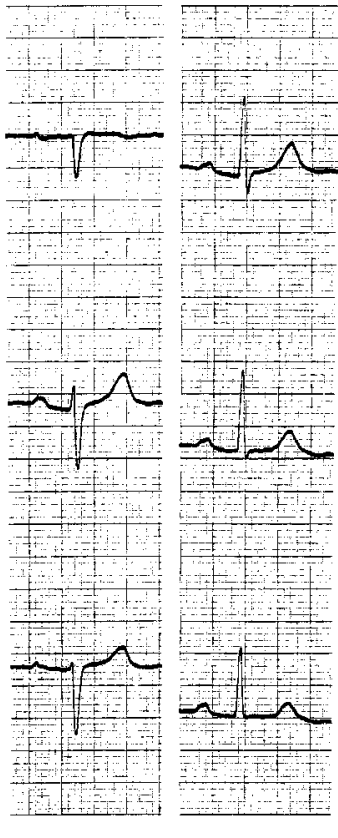

Figure 2 (A) ECG on admission to the intensive care unit for severe congestive heart failure. Severe ST-T alterations consistent with ventricular systolic overload are evident. (B) At one year follow up there is complete normality of the tracing, except for first degree atrioventricular block.

Fully evacuative pericardiocentesis produced $250 \mathrm{ml}$ of serous, haematic exudate negative for neoplastic cells and mycobacterium. A persistent butterfly-shaped erythemato-teleangectasic malar rash had been present since admission, therefore systemic lupus erythematosus was initially suspected, but laboratory tests and a search for specific autoantibodies proved negative. Pericardiocentesis and medical treatment resolved the symptoms. The patient was diagnosed with acute exudative pericarditis and discharged on maintenance treatment (diuretics, aspirin, antibiotic) and close follow up.

Unexpectedly she returned 11 days later because of worsening dyspnoea, peripheral oedema, cough, and recurrent evening fever. She was admitted to the intensive care unit owing to critical congestive heart failure. ECG showed worsening of ST-T changes consistent with ventricular systolic overload (fig 2A). A left posterobasal pleural effusion was evident on chest $x$ rays. Echocardiography showed no pericardial effusion but indicated severely reduced left ventricular systolic function (ejection fraction 26\%). Right heart Swan-Ganz monitoring showed raised central venous (34 $\mathrm{mm} \mathrm{Hg}$ ) and pulmonary wedge pressures $(36 \mathrm{~mm} \mathrm{Hg})$ and a reduced cardiac index $(2.5$ litres/min). Intravenous diuretics and nitrates, and oral angiotensin converting enzyme inhibitors led to rapid improvement, and the patient was moved from intensive care after a few days.

The patient had no history of intravenous drug abuse. Blood cultures were repeatedly negative. No endocardial vegetations were evident using transoesophageal echocardiography, but we could distinctly see diffuse thickening, shortening, and stiffening of the tricuspid leaflets (fig 3) without commissural
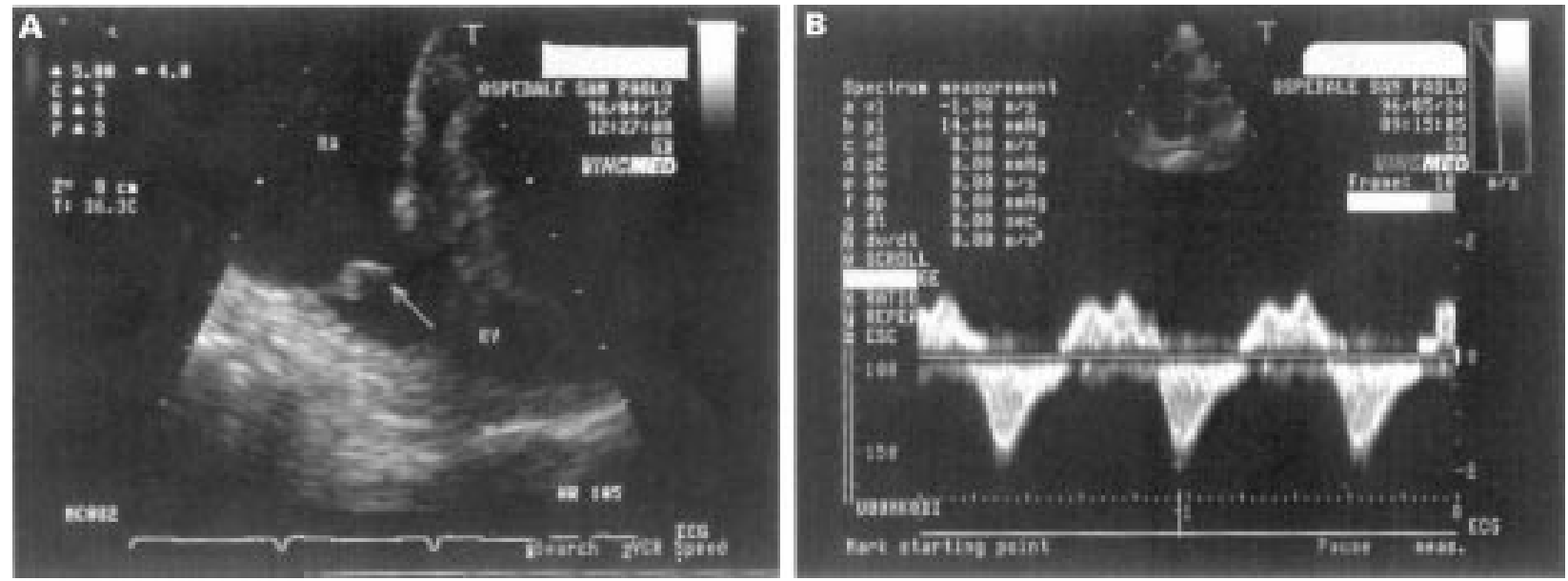

Figure 3 (A) Tricuspid valve aspect on transoesophageal echocardiography. (B) Typical dagger-shaped regurgitant signal of the tricuspid valve. Both suggest carcinoid heart disease. 
fusion or significant left sided valvar lesions; therefore, a rheumatic cause was improbable. Furthermore the continuous wave Doppler spectral profile of tricuspid regurgitation was dagger-shaped, with an early peak velocity and a rapid decline; the pressure half time was slightly prolonged. These aspects (just as described in a large echocardiography series) ${ }^{5}$ were consistent with the suspicion of carcinoid heart disease. Patency of the foramen ovale was excluded by transoesophageal echocardiography. The 24 hour urine 5-hydroxy indolacetic acid (5-HIAA) content was significantly raised $(45.0 \mathrm{mg} / 24 \mathrm{~h})$ and we subsequently observed several episodes of watery diarrhoea and flushing of the face and extremities. A fully expressed carcinoid syndrome was therefore confirmed-even the erythemato-teleangectasic malar rash could be considered typical, once systemic lupus erythematosus had been excluded.

Hepatic ultrasonography was done to localise the neoplasm: no metastases were found, excluding an origin from portal venous drainage tributary organs. No clinical or roentgenographic signs suggested a possible bronchial origin. Hence, looking for a rare site, we performed pelvic ultrasounds and found an $8 \mathrm{~cm}$, grossly rounded, left adnexial mass with a dishomogeneous cystic pattern. The report was confirmed on transvaginal echography and computed tomography (CT). No other lesions were found on total body CT.

The patient was treated with subcutaneous octreotide $150 \mu \mathrm{g}$ three times a day with complete resolution of diarrhoea, flushing, and cough.

Laparotomy tumour resection with total hysterectomy, bilateral adnexectomy, omentectomy, and appendectomy was carried out; no arrhythmias or haemodynamic complications occurred.

The ovarian mass was a large $(7.5 \times 7 \times 6 \mathrm{~cm})$, well encapsulated neoplasm of soft, elastic tissue with small cystic areas and a larger cavity $(3.5 \mathrm{~cm})$ containing hair and sebaceous material. No other malignant elements were found at surgical exploration of the abdominal cavity. Histology confirmed a low grade malignancy insular carcinoid in a mature ovarian cystic teratoma. Postoperative course was uneventful. Urinary 5-HIAA dosage before hospital discharge had returned to normal and the cardiac conditions were satisfactory.

At one year follow up the patient was asymptomatic on no medication. Urinary 5-HIAA was normal and there were no signs of malignancy or heart failure. Except for a first degree atrioventricular block (PR 0.24 seconds), the ECG is normal and shows pronounced reduction of a previously upper limit $\mathrm{P}$ wave voltage (fig $2 \mathrm{~B}$ ). A moderate tricuspid regurgitation with right atrial enlargement is the only abnormal echocardiographic finding; left ventricular function is normal. We believe the patient to be surgically healed from her malignancy, and no other measure except follow up of the tricuspidal disease is indicated.

\section{Discussion}

Up to $90 \%$ of carcinoids arise from the small bowel and the appendix. The remainder usually originate from other gastrointestinal sites or the bronchi. Ovary, thymus, and breast are very rare sites of origin. Carcinoid syndrome and carcinoid heart disease are caused by the offending action of many humoral substances produced by the tumour, usually inactivated by liver and lung enzymes. Gastrointestinal carcinoids tributary to portal vein circulation therefore give rise to carcinoid syndrome only when liver metastases secrete downstream from the hepatic filter. Ovarian carcinoids, on the contrary, release their products directly into the caval system and may give rise to carcinoid syndrome and carcinoid heart disease before the development of any metastatic lesion. Surgical removal can therefore be a radical treatment, and prevent the progression of heart lesions. For this reason prognosis of carcinoid heart disease from ovarian carcinoid tumours was considered good since the very first reports. ${ }^{4}$ Nevertheless other authors subsequently regarded the prognosis uncertain in the same cases ${ }^{6}$ and reported the possibility of progression of heart lesions despite surgical removal of the tumour. ${ }^{12}$ In such cases, cardiac surgery is indicated and can be life saving. ${ }^{2}$

In our case the patient presented with a myopericarditis-like clinical picture-not a typical presentation of carcinoid disease. The diagnosis was suspected on the basis of the tricuspid valve's peculiar aspect at ultrasound. Transoesophageal echocardiography was useful in magnifying this aspect, thus confirming the importance of both transthoracic and transoesophageal ultrasound in diagnosing carcinoid heart disease as already mentioned in literature. ${ }^{5} 78$

Pericarditis as a presentation of carcinoid heart disease was described in 1973 in one case of pericardial metastasis from a bronchial carcinoid tumour, ${ }^{9}$ but the pericardial effusion in that case was clearly related to a direct involvement from the contiguous neoplasm. A small pericardial effusion has been reported as a possible echocardiographic finding in carcinoid heart disease, and was present in $14 \%$ of cases in the largest recent series. ${ }^{5}$ Nevertheless, pericardial effusion remains an uncommon aspect of the disease and is an unexpected finding at echocardiography.

In contrast, we observed definite signs and symptoms of pericardial involvement as an initial manifestation of the disease, so that the diagnosis of acute pericarditis of unknown origin was initially formulated. Origin of the heart failure was also unclear. Right heart failure could have been related to the tricuspid valve disease whereas left heart failure had no evident causes. Moreover, left sided heart involvement in carcinoid heart disease is present in only $7 \%$ of patients ${ }^{5}$ and occurs in bronchial carcinoids or when a patent foramen ovale coexists, or perhaps with very prolonged and severe disease (a unique case requiring quadruple valve replacement has been described). ${ }^{10}$ Reduced left ventricular systolic 
function is described in fewer than $4 \%$ of the largest published series. ${ }^{5}$

We could therefore clinically suspect an accompanying or complicating myocarditis of unknown origin. The heart failure could otherwise be related directly to the carcinoid heart disease as hypothetical "toxic" damage by substances produced by the tumour. In this regard, an endomyocardial biopsy could have been interesting, but was deemed of no practical benefit in management and was not performed.

Finally, we emphasise the efficacy of octreotide in resolving all major symptoms of the carcinoid syndrome in our case, and we believe that the uncomplicated intraoperative course that ensued was largely the result of pretreatment with this long acting somatostatin analogue.

We gratefully acknowledge the gynaecological team of our hospital, particularly Dr Maria Ferrari, for surgical treatment; and the pathologists Dr Carlo Patriarca and Dr Solange Romagnoli for histological diagnosis.
1 Oei SG, Kloosterman MD, Verhoeven ATHM. Primary ovarian carcinoid tumour in combination with carcinoid heart disease; a case report. Eur $\mathcal{F}$ Obst Gynaec Repr Biol 1989;31:185-8.

2 Wilkowske MA, Hartmann LC, Mullany CJ, et al. Progressive carcinoid heart disease after resection of primary ovarian carcinoid. Cancer 1994;73:1889-91.

3 Watson JT, Badner NH, Ali MJ. The prophylactic use of octreotide in a patient with ovarian carcinoid and valvular heart disease. Can f Anaesth 1990;37:798-800.

4 Chatterjee K, Heather JC. Carcinoid heart disease from primary ovarian carcinoid tumours. A case report and review of literature. Am f Med 1968;45:643-8.

5 Pellika PA, Tajik AJ, Khandheria BK, et al. Carcinoid heart disease: clinical and echocardiographic spectrum in 74 patients. Circulation 1993;87:1188-96.

6 Stephan E, de Wit J. Carcinoid heart disease from primary carcinoid tumour of the ovary. Haemodynamic and cine coronary angiocardiographic study after operation. $\mathrm{Br}$ Heart f 1974;36:613-16.

7 Howard RJ, Drobac M, Rider WD, et al. Carcinoid heart disease: diagnosis by two-dimensional echocardiography. Circulation 1982;66:1059-65.

8 Lundin L, Landelius J, Andren B, et al. Transoesophageal echocardiography improves the diagnostic value of cardiac ultrasound in patients with carcinoid heart disease. $\mathrm{Br}$ ultrasound in patients
Heart $\mathcal{F}$ 1990;64:190-4.

9 Rich LL, Lisa CP, Nasser WK. Carcinoid pericarditis. Am $\mathcal{f}$ Med 1973;54:522-7.

10 Knott-Craig CJ, Schaff HV, Mullany CJ, et al. Carcinoid disease of the heart. Surgical management of ten patients. $f$ Thorac Cardiovasc Surg 1992;104:475-81. 